\title{
Antiferromagnetic Alignment and Relaxation Rate of Gd Spins in the High Temperature Superconductor $\mathrm{GdBa}_{2} \mathrm{Cu}_{3} \mathrm{O}_{7-\delta}$
}

\author{
R. J. Ormeno and C. E. Gough \\ School of Physics and Astronomy, The University of Birmingham, Edgbaston, Birmingham, B15 2TT, United Kingdom. \\ Guang Yang \\ Department of Metallurgy and Materials, The University of Birmingham, Birmingham, B15 2TT, United Kingdom.
}

\begin{abstract}
The complex surface impedance of a number of $\mathrm{GdBa}_{2} \mathrm{Cu}_{3} \mathrm{O}_{7-\delta}$ single crystals has been measured at 10, 15 and $21 \mathrm{GHz}$ using a cavity perturbation technique. At low temperatures a marked increase in the effective penetration depth and surface resistance is observed associated with the paramagnetic and antiferromagnetic alignment of the Gd spins. The effective penetration depth has a sharp change in slope at the Néel temperature, $T_{N}$, and the surface resistance peaks at a frequency dependent temperature below $3 \mathrm{~K}$. The observed temperature and frequency dependence can be described by a model which assumes a negligibly small interaction between the Gd spins and the electrons in the superconducting state, with a frequency dependent magnetic susceptibility and a Gd spin relaxation time $\tau_{s}$ being a strong function of temperature. Above $T_{N}, \tau_{s}$ has a component varying as $1 /\left(T-T_{N}\right)$, while below $T_{N}$ it increases $\sim T^{-5}$.
\end{abstract}

PACS numbers : 74.25.Ha, 74.72 -h, 74.25.Nf

One of the surprising early discoveries about high temperature superconductors was their apparent insensitivity to out-of-plane magnetic ions, with the superconducting properties of YBCO remaining almost unchanged when yttrium was replaced by magnetif rare earth ions, with the exception of $\mathrm{Ce}, \mathrm{Pr}$ and Tbl. Furthermore, the low temperature antiferromagnetic properties were observed to be independent of doping. The thermodynamic superconducting and antiferromagnetic properties of $\mathrm{GdBa}_{2} \mathrm{Cu}_{3} \mathrm{O}_{7-\mathrm{f}}(\mathrm{GBCO})$ therefore appear to be completely uncoupled 3 . However, there remains the possibility that the mutual interaction of the rare earth (RE) spins and the superconducting electrons could lead to changes in their dynamic properties. Interest in the antiferromagnetic alignment of the rare earth spins has also re-emerged as a likely explanation of the anomalous increase in the microwave surface resistance of GBCO thin films observed at low temperatures 16 .

We have therefore undertaken a systematic microwave investigation of the antiferromagnetic spin alignment in a number of GBCO single crystals. The surface impedance has been measured to well below the Gd Néel temperature $T_{N} \sim 2.2 \mathrm{~K}$, using a hollow dielectric resonator techniquet. Measurements at 10, 15 and $21 \mathrm{GHz}$ confirm the influence on microwave properties of the alignment of the Gd spins and enable us to determine the Gd spin relaxation time $\tau_{s}$ both above and below the Néel temperature.

In the superconducting state the microwave surface impedance is given by

$$
Z_{s}=\sqrt{\frac{\mathrm{i} \mu_{r} \mu_{0} \omega}{\left(\sigma_{1}-\mathrm{i} \sigma_{2}\right)}},
$$

where $\sigma_{1}$ is the normal state quasi-particle conductance $n_{q p} e^{2} \tau_{q p} / m$ ( $\tau_{q p}$ is the quasi-particle scattering time). In the ideal superconducting state $\sigma_{2}(T)=n_{s}(T) e^{2} / m \omega=$ $\left(\lambda(T)^{2} \mu_{0} \omega\right)^{-1}$, where $\lambda(T)$ is the penetration depth unperturbed by any coexistent magnetic properties and $n_{s}$ is the superfluid density. However, in the presence of magnetic spins $\sigma_{2}=\left(\lambda^{2} \mu_{r} \mu_{0} \omega\right)^{-1}$ and we can assume a relaxation model for a frequency dependent permeability, $\mu_{r}(T, \omega)=1+\chi(T) /\left(1+\mathrm{i} \omega \tau_{s}(T)\right)$, with a spin lattice relaxation time $\tau_{s}$. In this situation the field penetration is modified but not the intrinsic penetration depth which is related to the superfluid fraction. For a antiferromagnetic system above the Néel temperature $T_{N}$ we expect $\chi(T)=C /\left(T+T_{N}\right)$, with $C=n \mu_{B}^{2} p^{2} / 12 \pi k_{B}$, where $n$ is the number of spins per unit volume DC magnetic measurements give an effective moment $p=7.82$, in good agreement with the free ion value of 7.92 . Rewriting $Z_{s}$ as $\left[i \mu_{0} \omega / \sigma_{e f f}\right]^{1 / 2}$, we can define an effective conductivity as

$$
\sigma_{e f f}=\frac{\left(\sigma_{1}-\mathrm{i} \sigma_{2}\right)}{1+\chi /\left(1+\mathrm{i} \omega \tau_{s}\right)}
$$

The real and imaginary parts are give by

$$
\sigma_{1 e f f}=\frac{\left(1+\omega^{2} \tau_{s}^{2}\right)\left(\Gamma \sigma_{1}+\sigma_{2} \chi \omega \tau_{s}\right)}{\Gamma^{2}+\chi^{2} \omega^{2} \tau_{s}^{2}}
$$

and

$$
\sigma_{2 e f f}=\frac{\left(1+\omega^{2} \tau_{s}^{2}\right)\left(\sigma_{1} \chi \omega \tau_{s}-\Gamma \sigma_{2}\right)}{\Gamma^{2}+\chi^{2} \omega^{2} \tau_{s}^{2}}
$$

where $\Gamma=1+\omega^{2} \tau_{s}^{2}+\chi$. At low temperature, we will show that $\omega \tau_{s}>1$, so that

$$
\sigma_{1 e f f} \sim \sigma_{1}+\sigma_{2} \chi / \omega \tau_{s}
$$

and 


$$
\sigma_{2 e f f} \sim \sigma_{2}\left(1+\chi / \omega^{2} \tau_{s}^{2}+2 / \omega^{2} \tau_{s}^{2}\right)-\sigma_{1} \chi / \omega \tau_{s},
$$

where we retained terms to second order in $1 / \omega \tau_{s}$ because $\sigma_{2} \gg \sigma_{1}$.

In fitting our data we have also assumed no change in the quasi-particle conductance from the Gd spin fluctuations. To test the validity of this model, we have measured the surface impedance of several GBCO single crystals both above and below $T_{N}$ and at several microwave frequencies.

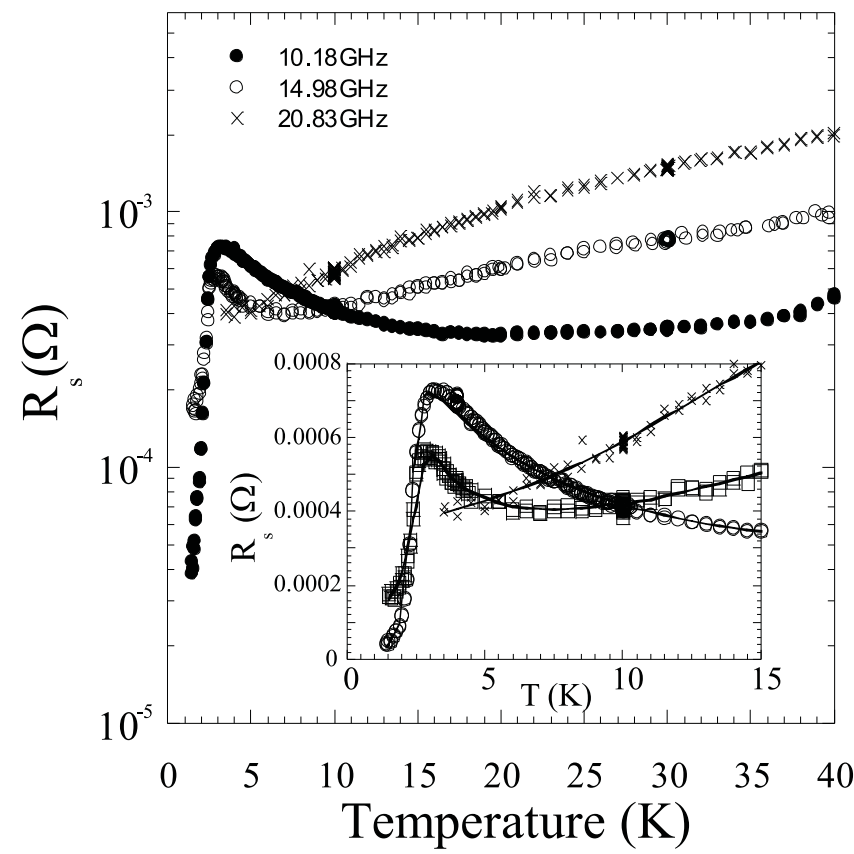

FIG. 1. The surface resistance $R_{s}(T)$ for currents flowing in the $a-b$ plane of a high quality $\mathrm{GdBa}_{2} \mathrm{Cu}_{3} \mathrm{O}_{7-\delta}$ single crystal. The inset shows an expanded view of the low temperature region. The solid lines represent calculated values of $R_{s}(T)$ using equation 3 , with values of $\chi(T)$ and $\tau_{s}$ derived from our model.

The GBCO crystals were orown in $\mathrm{BaZrO}_{3}$ crucibles to minimise contamination 9 , 10 . The largest crystal used in these measurements was $1.0 \times 1.1 \times 0.06 \mathrm{~mm}^{3}$ with a $T_{c}$ of $93 \mathrm{~K}$. During the course of the experiments, with the sample held overnight at room temperature under vacuum, the microwave transition at $T_{c}$ was observed to broaden, with a second transition emerging at $\sim 63 \mathrm{~K}$. This second transition is similar to earlier measurements by Srikanth et al. on a similarly grown YBCO fystal, which was interpreted as a second energy gap 11 . We believe the anomaly is more likely to be associated with oxygen diffusion out of the sample, resulting in surface regions of oxygen deficient, $60 \mathrm{~K}$ phase. This may be a generic problem for $\mathrm{BaZrO}_{3}$-grown HTS crystals held in vacuum at room temperature for any length in time. However, no associated changes were observed in the low temperature microwave properties in our measurements.
The surface impedance was measured using a cylindrical dielectric resonator with a $2 \mathrm{~mm}$ hole passing along its axis. The resonator was placed centrally within an OFHC copper cavity. Measurements were made using the $\mathrm{TE}_{01 n}$ resonant modes with a typical unloaded $Q$ values of $\sim 10^{5}$ at $10 \mathrm{GHz}$. The temperature of the dielectric resonator and copper cavity was held constant at the helium bath temperature. The sample was placed at a magnetic field antinode of the dielectric resonator, and was supported on the end of a long sapphire rod passing centrally through the resonator. The crystal could be heated from $1.2 \mathrm{~K}$ to well above $T_{c}$ by a heater mounted outside the cavity. Experiments were performed with the $c$-axis parallel or perpendicular to the rf magnetic field, to investigate the affect of magnetic anisotropy. From neutron diffraction experiments, the Gd spins are known to align along the $c$-axis 3 . Measurements were made at three of the resonant modes of the dielectric resonator close to 10,15 and $21 \mathrm{GHz}$ with suitable positioning of the sample.

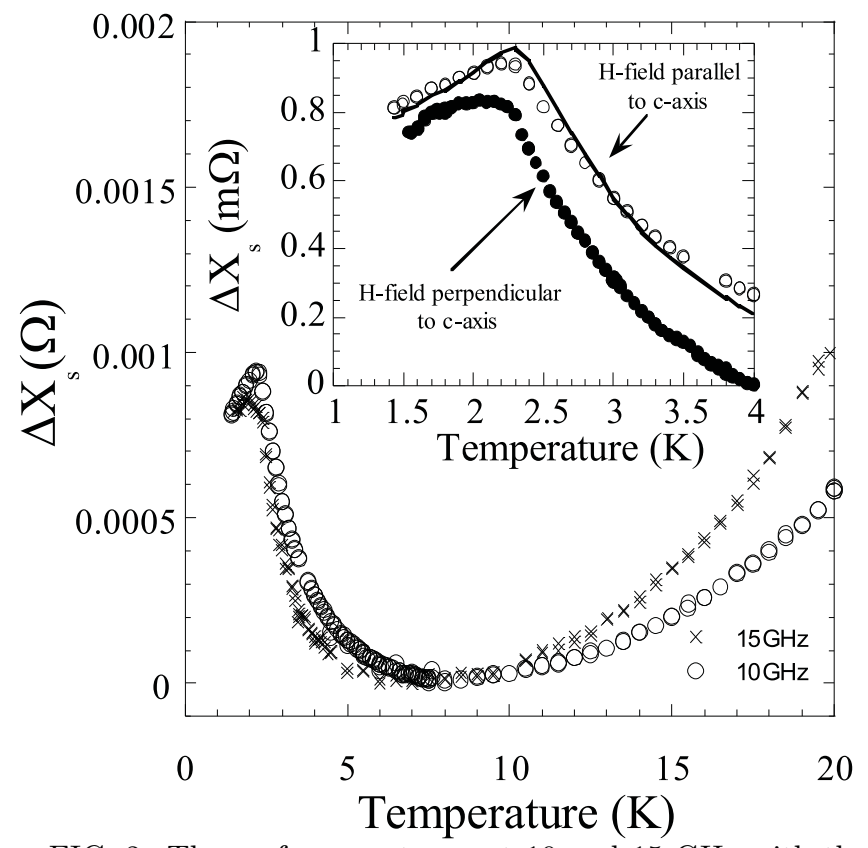

FIG. 2. The surface reactance at 10 and $15 \mathrm{GHz}$ with the $\mathbf{H}$-field parallel to the $c$-axis. The inset shows $\Delta X_{s}(T)$ for two crystal orientations at $10 \mathrm{GHz}$, the solid line represents a fit using equation 4 . Open circles are for the $\mathbf{H}$-field parallel to the $c$-axis and the closed circles for the field perpendicular to the $c$-axis.

The microwave properties were determined by a conventional cavity perturbation method using a HP $8722 \mathrm{C}$ Network analyser with additional data processing to obtain accurate measurements of the resonant frequency $f_{0}$ and half power bandwidth $f_{B}$ of the dielectric cavity resonances. The changes in these values with temperature can be related to the sur- 
face impedance by the cavity perturbation formula, $\Delta f_{B}(T)-2 \mathrm{i} \Delta f_{0}(T)=\Gamma\left(R_{s}+\mathrm{i} \Delta X_{s}\right)$. The resonator constant $\Gamma$ was determined from measurements with the sample replaced by a chemically polished niobium sample of the same size and known resistivity. We were able to achieve a measurement accuracy and reproducibility for $R_{s}$ of $\pm 20 \mu \Omega$ and an error in $\Delta \lambda= \pm 0.3 \AA$, for a sample of area $0.5 \times 1.1 \mathrm{~mm}^{2}$ at $10 \mathrm{GHz}$.

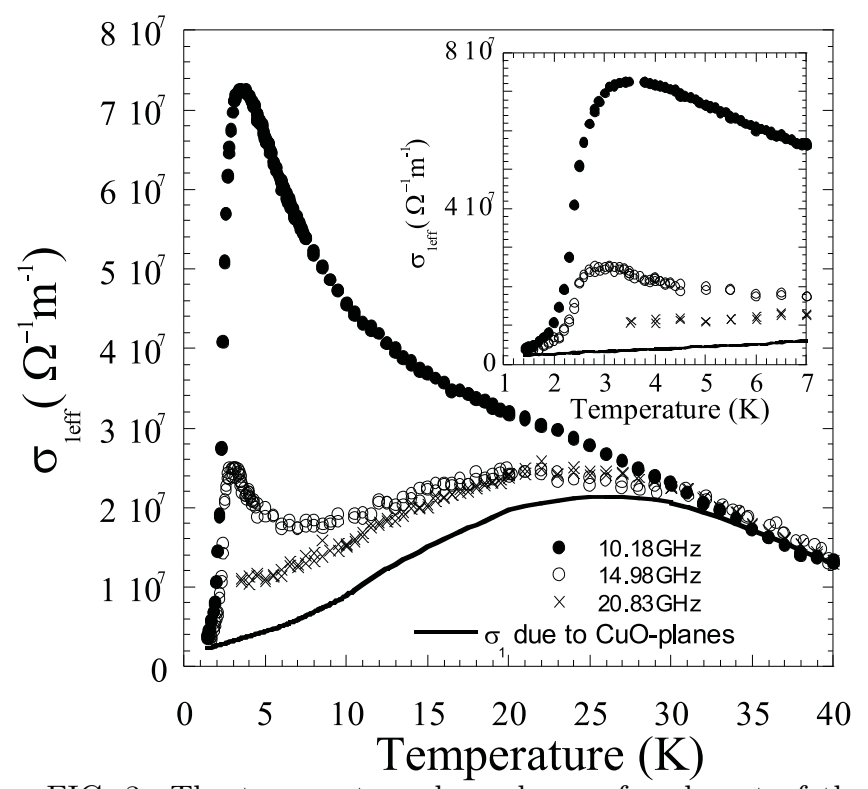

FIG. 3. The temperature dependence of real part of the microwave conductivity extracted from the data in figure 1 . The solid curve is $\sigma_{1}(T)$ due to the $\mathrm{Cu}-\mathrm{O}$ planes. The inset is an expanded view of the low temperature behaviour.

Figure 1 shows measurements of $R_{s}(T)$ at the three frequencies. For $T \geq 30 \mathrm{~K}$ the losses are quadratic in $\omega$, consistent with $\omega \tau_{q p} \ll 1$. In absolute terms, the losses are somewhat larger than observed in the best YBCO crystals, but are comparable with $R_{s}$ data for near optimally doped BSCCO crystals 12 . At lower temperatures there is a marked and strongly frequency dependent rise in losses, which we associate with the paramagnetic alignment of the Gd spins above the Néel temperature. The losses at $10 \mathrm{GHz}$ peak at $3.5 \mathrm{~K}$, significantly above the Néel temperature of $2.2 \mathrm{~K}$ (see inset of Figure 11). These losses then decrease by more than an order of magnitude at the lowest temperatures.

The spin alignment also leads to a small but pronounced increase in the reactive part of the surface impedance at low temperatures, as shown for two frequencies in figure 2. This corresponds to an increased penetration depth with a sharp change in slope defining the Néel temperature. Above $\sim 7 \mathrm{~K}$, the reactance increases as $\sim T^{2}$, in contrast to the $T$-dependence observed for high quality YBCO crystals. The magnitude of these losses and the $T^{2}$ dependence of the penetration depth imply a larger quasi-particle scattering than ob- served in optimally doped YBCO crystal 13 . Above $10 \mathrm{~K}$ we have the expected $\omega$ dependence of $X_{s}$, but at low temperatures $(T<7 \mathrm{~K})$ the reactance has a $\omega^{-1}$ dependence, as predicted by our model. The inset illustrates measurements with the crystal aligned with its $c$-axis parallel and perpendicular to the microwave magnetic field (open and closed circles respectively). In a parallel microwave field, the expected direction of spin alignment, the reactance drops linearly below a well-defined transition temperature $T_{N} \sim 2.25 \mathrm{~K}$, but in the perpendicular configuration the susceptibility below $T_{N}$ is much flatter and may even go through a small maximum. Similar results to those illustrated in Figures 11 and 2 were observed for all three single crystals investigated. The crystals included slightly underdoped, as-grown and close to optimum-doped oxygen annealed samples, from different growth batches.

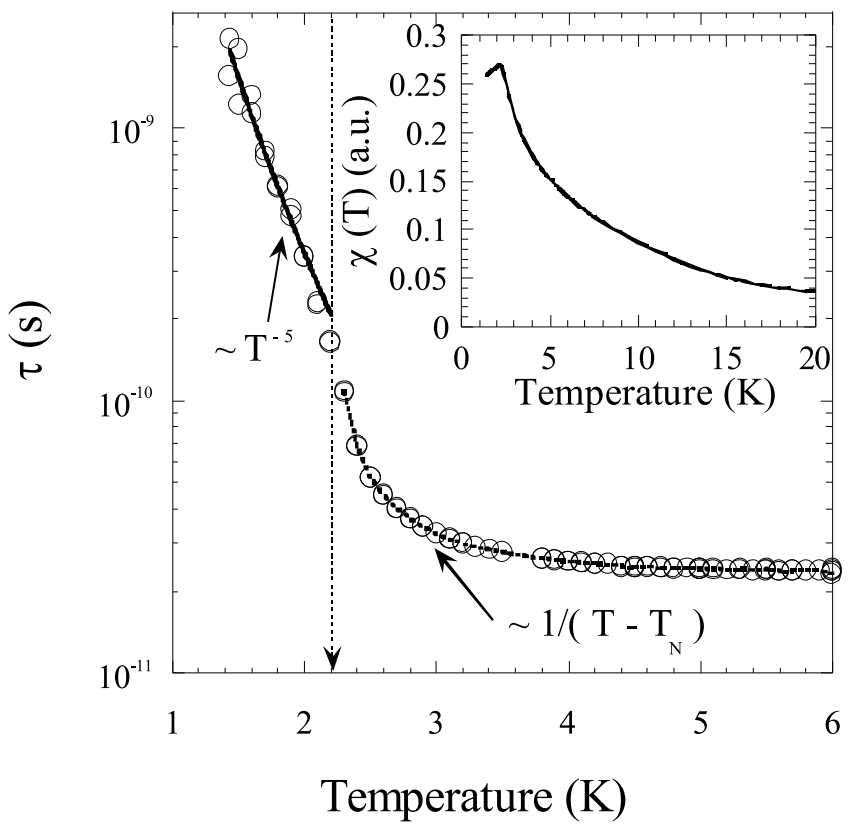

FIG. 4. The temperature dependence of the spin lattice relaxation time extracted from the $10 \mathrm{GHz}$ data . At the Néel temperature there is an apparent change in slope in $\tau_{s}(T)$. Above $T_{N}, \tau_{s}(T) \sim\left[T_{N} /\left(T-T_{N}\right)\right]^{\alpha}$ with the best fit obtained for $\alpha=1$, below $T_{N}$ the relaxation time varies as $\sim T^{-5}$. The inset demonstrates the temperature dependence of $\chi$ used to extract $\tau_{s}(T)$.

To extract an effective conductivity $\sigma_{1 e f f}$ from $R_{s}=\frac{1}{2} \omega^{2} \mu_{0}^{2} \sigma_{1 e f f} \lambda^{3}$, we have assumed: (i) a value for $\lambda(0)=140 \mathrm{~nm}$, typical of high quality YBCO samples (ii) above $10 \mathrm{~K} \sigma_{2 \text { eff }} \sim \sigma_{2}$, and (iii) below $10 \mathrm{~K}$ $\lambda(T) \sim T^{2}$ consistent with the $T^{2}$ temperature dependence of $X_{s}(T) \sim \omega \mu_{0} \lambda(T)$ in figure 2. We have also assumed that $\sigma_{1}$ is not significantly affected by the Gd spin fluctuations. Figure 3 shows the temperature dependence of the derived values of $\sigma_{1 e f f}$ for all three frequencies measured. Because the contribution to the effective 
conductivity from the Gd spins varies $\sim 1 / \omega \tau_{s}$, using the 10 and $15 \mathrm{GHz}$ data, we can extract $\sigma_{1}$ giving the solid line. The derived temperature dependence is similar to the variation of $\sigma_{1}(\mathrm{~T})$ observed in YBCO single crystals. It increases to a broad peak at about $25 \mathrm{~K}$, reflecting the increase in the quasi-particle scattering lifetime at low temperatures.

In our model, the additional losses when $\omega \tau_{s}>1$ are largely associated with the paramagnetic relaxation of the Gd spins in the microwave field. These losses, which we interpret as a decrease in the $\mathrm{Gd}$ spin relaxation rate on approaching and passing through the antiferromagnetic phase transition, peak at a frequency dependent temperature significantly above $T_{N}$. In this respect, we note that there is no significant change in $\sigma_{1 e f f}$ at the Néel temperature $T_{N}$ (see inset of figure 3). Any such affect is masked by the much larger changes in $\tau_{s}$, figure 4.

The region near $T_{N}$ might be expected to be dominated by antiferromagnetic spin fluctuations. We assume that close to $T_{N}, \tau_{s}$ involves a temperature dependent term varying as $\left[T_{N} /\left(T-T_{N}\right)\right]^{\alpha}$. A fit to this relation for $\alpha=1$ is shown in figure 4 . Below the antiferromagnetic transition $\tau_{s}$ increases by an order of magnitude varying $\sim T^{-5}$ with an apparent change in slope at $T_{N}$. The inset of figure 4 shows $\chi(T)$ used to derive the temperature dependence of $\tau_{s}$. This assumes a Curie-Weiss temperature dependence above $T_{N}$ and a slight drop in $\chi(T)$ below $T_{N}$ consistent with the measurement shown in the inset of figure 2 .

The model we have applied assumes that there is no interaction between the quasi-particles and spins (this is consistent with specific heat data on GBCO where no change is observed between semiconducting and superconducting samples2). Susceptibility measurements on non-superconducting GBCO have shown that $\chi(T)$ fits a 2-d Ising model above $T_{N} 14$. Below the transition $\chi(T)$ remains anomalously high, deviating from the Ising model. This is consistent with our reactance measurements, where we see only a small change in $\Delta X_{s}(T)$ below $T_{N}$, figure 2. This is in contrast to what is seen in other RE substitutions (Sm, Dy and $\mathrm{Nd}$ ) where the specific heat data capbe fitted to a 2-d Ising model both above and below $T_{N} 15$.

In the insets of figures 11 and 2, the solid lines fitted to the data have been evaluated using equations 3 and 4. We have assumed: (i) $\chi(T)=C /\left(T+T_{N}\right)$, with a value of $C=n \mu_{B}^{2} p^{2} / 12 \pi k_{B}$ corresponding to a derived magnetic moment $p=9.5$, slightly larger than deduced from magnetic measurement\& (ii) the derived Gd spin relaxation time plotted in figure 4 , and (iii) the complex electronic conductivity given by the derived value of $\sigma_{1}$ in figure 3 and a value of $\sigma_{2}$ assuming $\lambda(0)=140 \mathrm{~nm}$. The excellent fit to the experimental data supports our theoretical model. In particular, there appears to be no need to invoke any additional effects, such as a modification of the electronic mean free path from the Gd spin fluctuations.
In summary we have presented extensive microwave surface impedance measurements on GBCO single crystals at several frequencies to investigate the influence of the antiferromagnetic alignment of the $\mathrm{Gd}$ spin at low temperatures. We are able to describe the experimental results by a model involving the increase in magnetic susceptibility associated with antiferromagnetic alignment and a strongly temperature dependent relaxation time. The derived spin lattice relaxation time increases below $T_{N}$ with a temperature dependence $\sim T^{-5}$. Above $T_{N}$, $\tau_{s} \sim 1 /\left(T-T_{N}\right)$. Within the accuracy of our measurements, $\sigma_{1}(T)$ is not affected by the antiferromagnetic alignment of the $\mathrm{Gd}$ spins.

We thank G. Walsh and D. Brewster for valuable technical support. We also thank A. Porch and M. Hein for useful discussions. This research is supported by the EPSRC, UK.

${ }^{1}$ P. H. Hor et al., Phys. Rev. Lett. 58, 1891 (1987).

${ }^{2}$ B. D. Dunlap et al., Phys. Rev. B 37, 592 (1987).

${ }^{3}$ H. A. Mook et al., Phys. Rev. B 38, 12008 (1988).

${ }^{4}$ J. R. Waldram, Private communication.

${ }^{5}$ S. M. Anlage, Lucia Mercaldo, and Vladimir Talanov, Private communication.

${ }^{6}$ J. J. Wingfield, PhD Thesis, The University of Birmingham (unpublished) (1999).

7 J. J. Wingfield, J. R. Powell, C. E. Gough, and A. Porch, IEEE Trans. Appl. Supercon. 7, 2009 (1997).

${ }^{8}$ J. R. Thompson et al., Phys. Rev. B 37, 9395 (1988).

${ }^{9}$ Ruixing Liang, D. A. Bonn, and W. N. Hardy, Physica C 304, 105 (1998).

${ }^{10}$ A. Erb, E. Walker, and R. Flukiger, Physica C 245, 9 (1996).

${ }^{11}$ H. Srikanth et al., Phys. Rev. B 55, R14733 (1997).

${ }^{12}$ S. F. Lee et al., Phys. Rev. Lett. 77, 735 (1996).

13 D. A. Bonn et al., Phys. Rev. B 50, 4051 (1994).

${ }^{14}$ J. van de Berg et al., Solid State Communications 64, 699 (1987).

${ }^{15}$ K. N. Yang et al., Phys. Rev. B 40, 10963 (1989). 